\title{
Trends in Breast Measurements of Unilateral Breast Reconstruction Patients to Inform Bra Design
}

\author{
Krista M. NICKLAUS ${ }^{1,2}$, Eloise JEWETT'1 ${ }^{1}$ Chi LIU ${ }^{3}$, Jun LIU², Gregory P, REECE², \\ Summer E. HANSON ${ }^{2}$, Fatima A. MERCHANT ${ }^{1,4}$, Mia K. MARKEY*1,5 \\ ${ }^{1}$ Department of Biomedical Engineering, The University of Texas at Austin, Austin, TX, USA; \\ 2 Department of Plastic Surgery, The University of Texas MD Anderson Cancer Center, Houston, TX, USA; \\ ${ }^{3}$ Apparel and Art Design College, Xi'an Polytechnic University, Xi'an, Shannxi, P.R. China; \\ ${ }^{4}$ Department of Engineering Technology, University of Houston, Houston, TX, USA \\ ${ }^{5}$ Department of Imaging Physics, The University of Texas MD Anderson Cancer Center, Houston, TX, USA
}

https://doi.org/10.15221/19.272

\begin{abstract}
Breast reconstruction patients face unique challenges in finding a properly fitting bra after breast surgery, leading to decreased bra comfort and psychosocial functioning. In addition to considerations such as location of seams and choice of fabric, identifying trends in how breast shape and symmetry measurements change between native breasts and reconstructed breasts may help inform bra design for reconstruction patients. We have previously developed a correspondence system between bra measurements and clinical breast measurements used by reconstructive surgeons. The selected measurements describe the size and projection of the breasts as well as their relative location on the torso as captured by clinical photographs. In this study, we explore how reconstruction changes breast measurements pertinent to bra design by analyzing 3D surface torso images of 15 unilateral implantbased breast reconstruction patients before and after their reconstruction surgery. Using custom software developed at University of Houston, two researchers measured several breast properties on a 3D surface torso image taken before mastectomy and on another image taken after each patient's final implants had been placed. 14 of the 15 patients had completed their reconstruction surgeries by the time that the second image was taken. We compared the differences in measurements between the pre-mastectomy image and post-mastectomy and reconstruction image for both breasts separately, as well as change in right-left symmetry of the measurements between the pre- and post- images. 14 out of the 15 patients had a revision surgery performed on the contralateral breast to enhance postreconstruction symmetry. The three most affected measurements between native breasts and breasts reconstructed after mastectomy were the sternal notch to most projecting point, lateral point to most projecting point, and mid-clavicle to transition point to most projecting point. These changes can be attributed to the size and shape of the implants used to compensate for the removal of native breast tissue, which change the fundamental footprint and curvature of the breast. Asymmetry between the breasts also increased after breast cancer treatment despite reconstruction and contralateral revision procedures. These measurements can be used to inform bra designers of what adjustments may be needed to bra patterns to improve fit for reconstruction patients.
\end{abstract}

Keywords: Apparel design, Breast reconstruction, 3D body images

\section{Introduction}

Breast reconstruction is the process of surgically reforming a breast mound after mastectomy to achieve the look of a breast shape when clothed. The new breast mound can be reconstructed using either autologous tissue, such as tissue from the patient's abdomen or back, implants, or a combination of autologous tissue and implants. A reconstructed breast does not usually retain the same size, shape, or physical characteristics as a patient's native breasts, leading to new challenges in bra fit and comfort. Challenges with finding a properly fitting and comfortable bra can profoundly impact patients' quality of life and psychosocial functioning [1]. Breast cancer patients have expressed many unmet needs regarding their intimate apparel after breast cancer treatment and surgery. For example, seams play a major role in bra comfort, as seams that lay over scars, radiated skin, or swollen areas of the breast and torso can result in pain. Breast asymmetry affecting bra fit is another challenge that is amplified for women who undergo a mastectomy, even if followed by reconstructive surgery. While most women have some amount of breast asymmetry [2], mastectomy followed by reconstruction often results in dramatic changes in the volume and in the fundamental footprint (location on the chest wall and shape) of the breast, so that the bra can no longer properly support and protect the breasts.

\footnotetext{
* mia.markey@utexas.edu; +1-512-471-1711; bmil.bme.utexas.edu
} 
In prior work, we proposed a correspondence system between measurements used for bra design and measurements used in clinical practice on clinical photographs of breast reconstruction patients [3]. The goal of this study was to assess the anatomical changes between native breasts and reconstructed breasts and relate those changes to bra measurements for a patient population who underwent mastectomy followed by unilateral implant-based reconstruction. Implant-based reconstruction is the most common form of breast reconstruction in the United States [4], and women who have unilateral procedures may have unique challenges in bra fitting.

\section{Materials and Methods}

\subsection{Participants}

We have previously collected 3D surface torso images, medical record data, and psychosocial measures from a cohort of 505 patients who underwent mastectomy followed by breast reconstruction at The University of Texas MD Anderson Cancer Center from 2011-2014. 3D surface torso images were taken with a 3dMDTorso system (3dMD Inc., Atlanta, GA, USA) over various time points from before reconstruction to $18+$ months post reconstruction. For this study, we selected a group of patients who underwent unilateral implant-based breast reconstruction for whom images before reconstruction and after reconstruction were available. Measurements were made on both breasts on a pre-mastectomy image when the patient had native breasts and on a post-reconstruction image taken at least three months after the patient had a permanent implant placed after breast cancer treatment (mastectomy). Contralateral symmetry surgery (when the opposite breast is surgically changed to be closer in size to the reconstructed breast) is common practice and as such is depicted in some of the post-reconstruction images. Figure 1 provides examples of the participants' pre-mastectomy and post-reconstruction images.

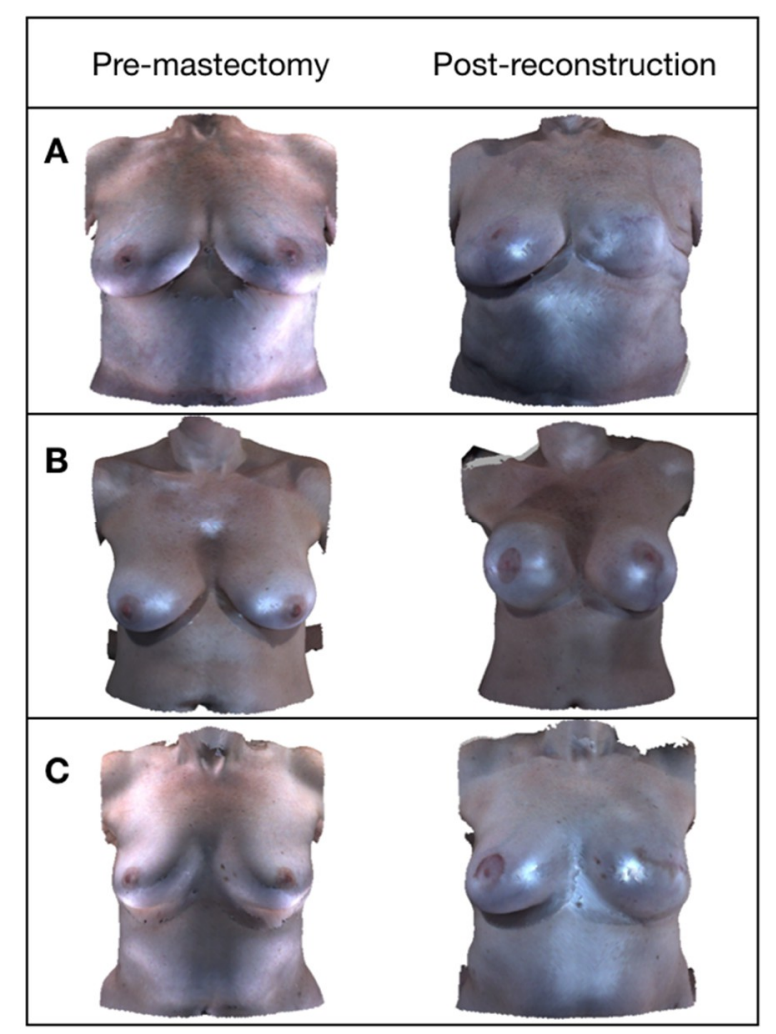

Fig. 1. Three examples of pre-mastectomy (left) and post-reconstruction (right) images for patients who received unilateral implant-based breast reconstruction to rebuild the breast mound after mastectomy. A) Patient received unilateral left implant reconstruction. The post-reconstruction image was taken 9 months after round implant placement, and the right breast received reduction and mastopexy revision procedures. B) Patient received unilateral right implant reconstruction. The post-reconstruction image was taken 3 months after round implant placement, and the left breast received augmentation and mastopexy revision procedures. C) Patient received unilateral left implant reconstruction. The post-reconstruction image was taken 3 months after round implant placement, and the right breast received augmentation and mastopexy revision procedures. 


\subsection{Measurements}

We previously identified seven measurements on clinical photographs that correspond to measurements typically used for bra design [3], which define the size and contours of the breast, as well as the relative location of the breasts on the torso. Since reconstructed breasts often do not have nipples, we defined the bust point as the most projecting point of the breast. Definitions and visual representations of the measurements are displayed in Figure 2.

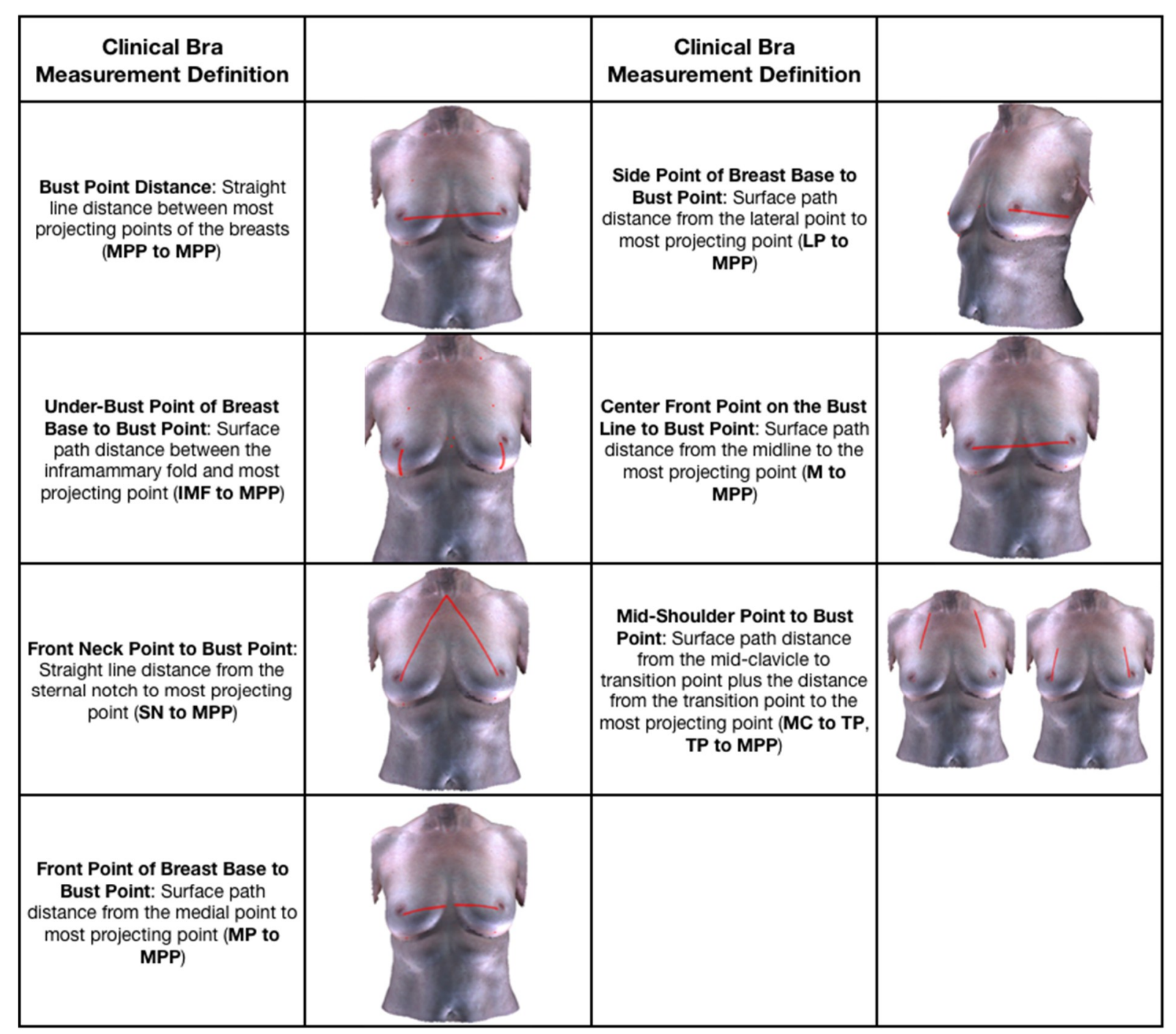

Fig. 2. Corresponding measurements between bra design and clinical practice

The seven measurements were made with custom software that allows for viewing, manipulating, and measuring 3D images [5]-[9]. Two trained researchers (KN and EJ) manually selected the eight required fiducial points (Most Projecting Point of the breast (MPP), Inframammary Fold at the meridian (IMF), Sternal Notch (SN), Medial Point - most medial point at the end of the IMF (MP), Lateral Point most lateral point at the end of the IMF (LP), Midline (M), Mid-clavicle (MC), Transition Point - post of transition from the chest wall to the breast on the meridian line (TP)) when present on each image, which were verified by an experienced reconstructive surgeon (GR). In addition to the surface path measurements, the 3D partial Breast Retraction Assessment [7] and breast volumes were calculated for each image with the same software as secondary analyses of symmetry. Breast volume was calculated [8] using the surface area defined by four manually-selected points: sternal notch, anterior axillary, lateral point below the level of the lowest visible point, and midline below the level of the lowest visible point. 


\section{Results}

\subsection{Participant Demographics}

Fifteen patients met the criteria of having a unilateral implant-based reconstruction and available premastectomy and post-implant placement images. All of the participants had a skin-sparing mastectomy for their breast cancer treatment, and a two-stage implant-based reconstruction, where their initial reconstruction procedure involved placing a temporary tissue expander that was exchanged in a later surgery for a permanent implant. Implant sizes ranged from $300 \mathrm{ml}$ to $1000 \mathrm{ml}$. Fourteen of the fifteen participants had a contralateral symmetry procedure performed before the post-implant placement images were taken, in order to visually match the non-reconstructed breast to the reconstructed breast. Twelve participants had completed their reconstruction procedures; three underwent additional procedures after the analyzed time point (fat grafting and implant size change). Participant demographics are presented in Table 1.

Table 1. Participant Demographics

\begin{tabular}{|c|c|}
\hline Variables & Statistics \\
\hline $\mathrm{N}$ & 15 \\
\hline Age (years) & $\begin{array}{l}\text { Mean } \pm S D: 48 \pm 10 \\
\text { Range: } 35-66\end{array}$ \\
\hline $\mathrm{BMI}\left(\mathrm{kg} / \mathrm{m}^{2}\right)$ & $\begin{array}{l}\text { Mean } \pm \text { SD: } 28.0 \pm 6.9 \\
\text { Range: } 21.4-41.1\end{array}$ \\
\hline $\begin{array}{l}\text { Diagnosis } \\
\text { DCIS } \\
\text { IDC }\end{array}$ & $\begin{array}{l}8(53 \%) \\
7(47 \%)\end{array}$ \\
\hline $\begin{array}{l}\text { Mastectomy Type } \\
\text { Skin-sparing } \\
\text { Nipple-sparing }\end{array}$ & $\begin{array}{l}15(100 \%) \\
0(0 \%)\end{array}$ \\
\hline $\begin{array}{c}\text { Reconstruction Laterality } \\
\text { Right breast } \\
\text { Left Breast }\end{array}$ & $\begin{array}{l}9(60 \%) \\
6(40 \%)\end{array}$ \\
\hline $\begin{array}{l}\text { Implant Shape } \\
\text { Round } \\
\text { Anatomical } \\
\text { Unknown } \\
\end{array}$ & $\begin{array}{l}11(73 \%) \\
3(20 \%) \\
1(7 \%) \\
\end{array}$ \\
\hline $\begin{array}{l}\text { Contralateral Symmetry Procedures } \\
\text { Mastopexy } \\
\text { Reduction } \\
\text { Augmentation } \\
\text { Mastopexy, Reduction } \\
\text { Mastopexy, Augmentation } \\
\text { None }\end{array}$ & $\begin{array}{l}6(40 \%) \\
3(20 \%) \\
2(13 \%) \\
1(7 \%) \\
2(13 \%) \\
1(7 \%)\end{array}$ \\
\hline $\begin{array}{l}\text { Time between tissue expander } \\
\text { placement and implant placement } \\
\qquad \begin{array}{l}6 \text { months } \\
9 \text { months } \\
12 \text { months }\end{array} \\
\end{array}$ & $\begin{array}{l}9(60 \%) \\
3(20 \%) \\
3(20 \%)\end{array}$ \\
\hline
\end{tabular}

\subsection{Bra Measurements}

The inframammary fold fiducial point was not visible in the 3D clinical photograph for $40 / 60$ breasts, as ptotic breasts often obscure the inframammary fold. The IMF to MPP measurement was subsequently removed from the analyses. The locations of the lateral point and mid-clavicle point could not be identified for two patients due to holes in the images. Table 2 presents the summary statistics of the percent change between the pre-mastectomy image and post-reconstruction image for the reconstructed breasts and revised contralateral breasts. Percent change was chosen as the metric in order to compensate for the different breast sizes and shapes of the patients and was calculated with equation 1.

$$
\text { Percent Change }=100 * \frac{\text { Post-Pre }}{\text { Avg(Post }, \text { Pre })}
$$


The sign of the percent change was retained in order to see the direction of change (i.e., whether the distance increased or decreased).

Table 2. Summary statistics of the percent change between pre-mastectomy and post-reconstruction measurements for the reconstructed and contralateral breasts

\begin{tabular}{|c|c|c|c|c|c|c|c|c|}
\hline & \multicolumn{8}{|c|}{ Reconstructed Breasts } \\
\hline & $\begin{array}{c}\text { MPP to } \\
\text { MPP } \\
\text { (N=15 } \\
\text { breasts) }\end{array}$ & $\begin{array}{l}\text { SN to MPP } \\
(N=15)\end{array}$ & $\begin{array}{l}\text { MP to } \\
\text { MPP } \\
(N=15)\end{array}$ & $\begin{array}{l}\text { LP to MPP } \\
(\mathrm{N}=15)\end{array}$ & $\begin{array}{l}M \text { to MPP } \\
(N=15)\end{array}$ & $\begin{array}{c}M C \text { to } T P \\
(N=15)\end{array}$ & $\begin{array}{l}\text { TP to MPP } \\
(\mathrm{N}=15)\end{array}$ & $\begin{array}{l}M C \text { to TP } \\
\text { to MPP } \\
(\mathrm{N}=15)\end{array}$ \\
\hline $\begin{array}{l}\text { Mean } \\
\text { (SD) }\end{array}$ & $-5.5(11.9)$ & $-14.5(9.7)$ & $\begin{array}{l}-13.9 \\
(22.2)\end{array}$ & $\begin{array}{l}-13.0 \\
(21.5)\end{array}$ & $\begin{array}{l}-12.5 \\
(19.7)\end{array}$ & $\begin{array}{l}-21.5 \\
(13.5)\end{array}$ & $-0.2(19.1)$ & $\begin{array}{l}-11.6 \\
(10.5)\end{array}$ \\
\hline \multirow[t]{3}{*}{$\begin{array}{l}\text { Median } \\
\text { (IQR) }\end{array}$} & $-3.8(16.6)$ & $-14.7(5.8)$ & $\begin{array}{l}-12.9 \\
(26.6) \\
\end{array}$ & \begin{tabular}{|l|}
-16.4 \\
$(21.0)$ \\
\end{tabular} & $\begin{array}{l}-13.9 \\
(20.5) \\
\end{array}$ & $\begin{array}{l}-21.6 \\
(15.1) \\
\end{array}$ & $-1.4(22.0)$ & $\begin{array}{l}-11.1 \\
(10.8) \\
\end{array}$ \\
\hline & \multicolumn{8}{|c|}{ Contralateral Breasts } \\
\hline & $\begin{array}{c}\text { MPP to } \\
\text { MPP } \\
\text { (N=15 } \\
\text { breasts) }\end{array}$ & $\begin{array}{l}\text { SN to MPP } \\
(N=15)\end{array}$ & $\begin{array}{l}\text { MP to } \\
\text { MPP } \\
(N=15)\end{array}$ & $\begin{array}{l}\text { LP to MPP } \\
(\mathrm{N}=14)\end{array}$ & $\begin{array}{l}\text { M to MPP } \\
(\mathrm{N}=15)\end{array}$ & $\begin{array}{l}M C \text { to TP } \\
(N=14)\end{array}$ & $\begin{array}{l}\text { TP to MPP } \\
(\mathrm{N}=15)\end{array}$ & $\begin{array}{c}M C \text { to TP } \\
\text { to MPP } \\
(\mathrm{N}=14)\end{array}$ \\
\hline $\begin{array}{c}\text { Mean } \\
(\mathrm{SD})\end{array}$ & $-5.5(11.9)$ & $-6.9(11.0)$ & $-4.4(16.8)$ & $4.3(15.9)$ & $-6.1(15.4)$ & $0.2(5.6)$ & $\begin{array}{l}-13.5 \\
(25.6) \\
\end{array}$ & $-7.1(10.3)$ \\
\hline $\begin{array}{l}\text { Median } \\
\text { (IQR) }\end{array}$ & $-3.8(16.6)$ & $-5.9(13.3)$ & $-3.9(18.8)$ & $8.2(22.2)$ & $-9.1(13.7)$ & $0.2(8.6)$ & $-7.7(37.5)$ & $-6.2(12.7)$ \\
\hline
\end{tabular}

There was a significant difference between the percent change of the reconstructed breast and contralateral breast for SN-MPP (Student's paired two-sided t test, $p<0.001)$, LP-MPP $(p<0.05)$, and MC to TP to MPP $(p<0.05)$. When the MC to TP to MPP measurement is divided into the MC to TP and TP to MPP segments, the MC to TP measurement has the greatest percent change and the most significant difference between the reconstructed breast and contralateral breast $(p<0.0001)$. Figure 3 displays a boxplot of the percent change in the SN-MPP, LP-MPP, and MC-TP measurements between the pre-mastectomy and post-reconstruction time points for the reconstructed and contralateral breasts. These results demonstrate that a degree of asymmetry persists after reconstruction and contralateral symmetry procedures that must be accounted for.

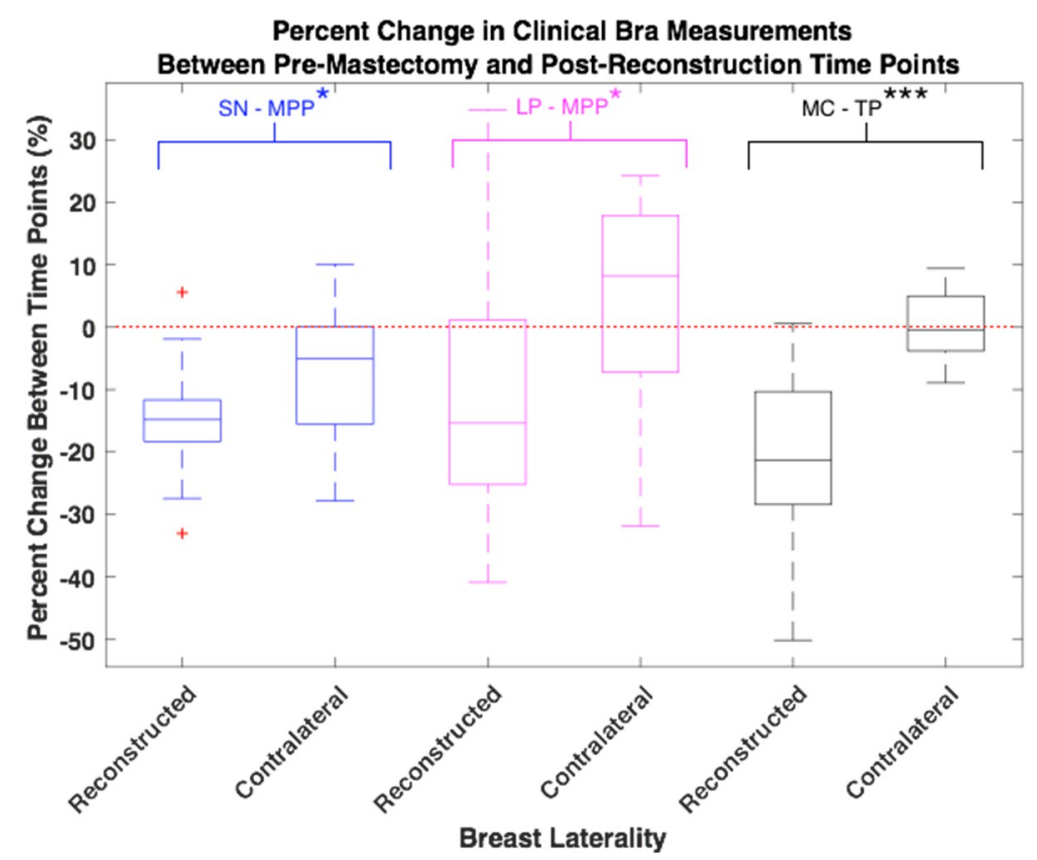

Fig. 3. Boxplot to demonstrate differences in three clinical bra measurements: SN-MPP, LP-MPP, and MC-TP between the reconstructed and contralateral breasts. The red dashed line indicates $0 \%$ change in the measurement between pre-mastectomy and post-reconstruction. For all three measurements presented, the reconstructed breast has a larger degree of change than the contralateral breasts. 


\subsection{Secondary Symmetry Analyses}

\subsubsection{D Percentage Breast Retraction Assessment (3D pBRA)}

The pBRA is a measure of symmetry that encompasses the differences between the horizontal position of the breasts in relation to the midline and vertical position of the breasts in relation to the level of the SN [10]. The pBRA ranges from 0 (perfect symmetry) and 1. 3D variant of pBRA was introduced and validated for 3D images by Kawale et al [7]. Larger values of 3D pBRA indicate more asymmetry. This symmetry measure requires identification of the mid-clavicle point, and was therefore unavailable for one image in our study. Table 3 contains the 3D pBRA values for each participant in the pre-mastectomy and post-reconstruction images. The median 3D pBRA in the pre-reconstruction images was 0.05 and 0.11 in the post-reconstruction images. There is a significant difference in symmetry between time points (Wilcoxon signed rank test, $p=0.025$ ). Figure 4 displays a boxplot of the 3D pBRA data.

Table 3. Summary statistics of 3D pBRA symmetry measure at the pre-mastectomy and post-reconstruction time points

\begin{tabular}{|c|c|c|c|}
\hline & \multicolumn{3}{|c|}{ 3D pBRA } \\
\hline & Pre-mastectomy & Post-reconstruction & Difference (Post-Pre) \\
\hline Mean (SD) & $0.07(0.11)$ & $0.14(0.12)$ & $0.07(0.17)$ \\
\hline Median (IQR) & $0.05(0.04)$ & $0.11(0.10)$ & $0.06(0.10)$ \\
\hline
\end{tabular}

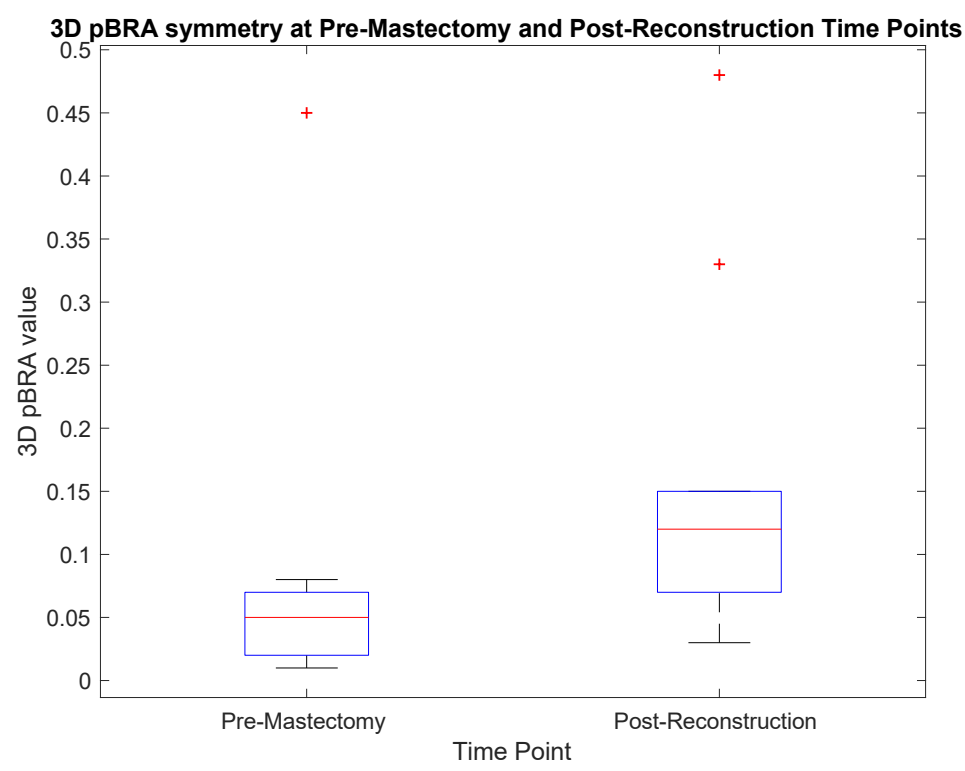

Fig. 4. Boxplot demonstrating the range of $p B R A$ values in the pre-mastectomy and post-reconstruction images. Larger values of $3 D$ pBRA indicate more asymmetry. Hence, mastectomy introduces considerable breast asymmetry that typically persists to some degree after reconstruction.

\subsubsection{Breast Volume Symmetry}

Breast volumes at both time points were calculated for $13 / 15$ participants. The values ranged from 252 $\mathrm{ml}$ to $1277 \mathrm{ml}$. Table 4 contains the absolute volume symmetry (absolute difference between the right and left breast volumes) at the pre-mastectomy and post-reconstruction time points. There is a significant increase in the magnitude of the volume asymmetry between the time points (Wilcoxon signed rank test, $p=0.01$ ). 
Table 4. Summary statistics of volume symmetry measure at the pre-mastectomy and post-reconstruction time points

\begin{tabular}{|c|c|c|c|}
\hline & \multicolumn{3}{|c|}{ Absolute Volume Symmetry between Left and Right Breasts } \\
\hline & Pre-mastectomy & Post-reconstruction & Difference \\
\hline Mean (SD) & $49.5(64.0)$ & $164.1(155.4)$ & $146.5(160.3)$ \\
\hline Median (IQR) & $33.8(41.9)$ & $100.1(189.3)$ & $66.4(182.6)$ \\
\hline
\end{tabular}

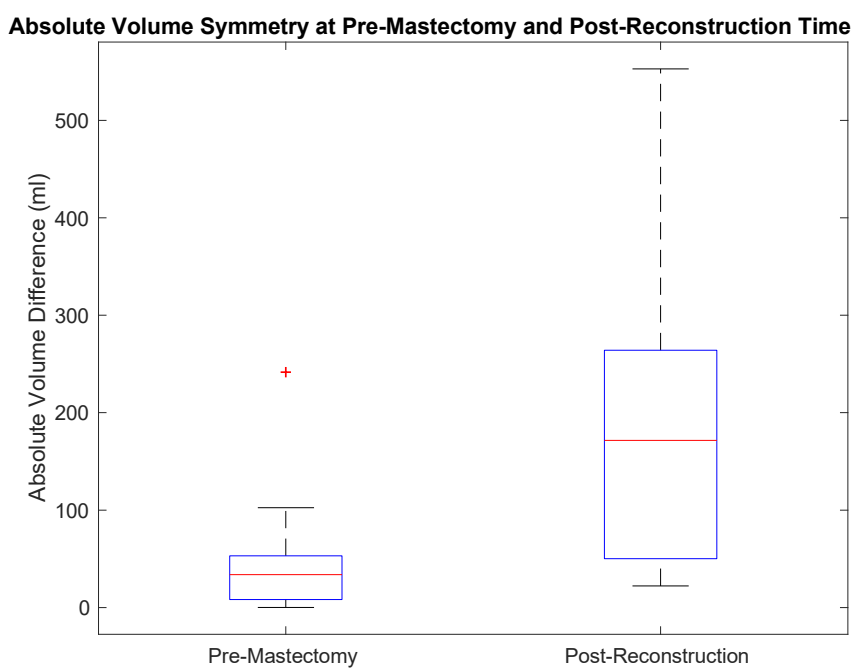

Fig. 5. Boxplot of the absolute volume symmetry between the breast affected by cancer and contralateral breast at the pre-mastectomy and post-reconstruction time points. Even after breast reconstruction to rebuild the breast mound, there is more volume difference between the right and left breasts after breast cancer treatment (mastectomy) than there is prior to treatment.

\section{Discussion}

The clinical bra measurements that were most affected by mastectomy followed by implant reconstruction surgery were the mid-clavicle (MC) to transition point (TP) to most projecting point (MPP) (driven by MC to TP), sternal notch (SN) to MPP, and lateral point (LP) to MPP measurements (see Figure 1 for measurement definitions). The movement of the MPP can come from two factors. First, a reconstructed breast often has a volume different from the native breast, which is a function of the implant thickness. In addition, for the patients who received a round implant, the shape of the implant is semi-spherical, so that the most projecting point is generally in the center of the implant. The most projecting point on a native breast will depend on the amount of breast ptosis, the distribution of tissue inside the breast, and the curvature of the breast and chest wall. If a person has ptotic, sagging breasts, the most projecting point will likely have greater vertical distance from the level of the sternal notch than someone with less ptotic breasts (either naturally or due to the presence of an implant). The change in the MP to TP and LP to MPP measurements is driven by the semi-spherical shape of the implant. The transition point is the point where the contour of the chest wall transitions to the breast. When a round implant is placed, the transition point will be approximately the upper edge of the implant with a sudden change in curvature, versus a native breast when the curvature of the chest wall gradually changes to indicate the upper curvature of the breast. The transition point of the reconstructed breast will also depend on the thickness of the soft tissue overlaying the implant. The lateral point is where the inframammary fold ends as the curvature of the breast ends laterally. After implant reconstruction, the lateral point is mostly determined by the diameter of the implant, which can cause the lateral point to either move more medial or lateral depending on the native breast footprint and size of implant. In our patient population, the lateral point tended to move more medial than the native breast lateral point. 
Similar trends in the measurement changes occurred for the revised contralateral breasts, but to a lesser extent than for the reconstructed breasts. The MPP also tends to move upwards relative to the level of the sternal notch in procedures such as a breast augmentation and mastopexy (breast lift). In contrast to the reconstructed breasts, the LP to MPP measurements increased for the revised contralateral breasts, as the fullest part of the breast is moved vertically, which can widen the breast footprint (and also raising the TP). For 8/13 of the participants, the revised contralateral breast was larger than the reconstructed breast, which also increases the LP to MPP distance.

The key implication for bra design for this patient population is that the curvature of an implant-based reconstructed breast is more rigid and dramatic than that of the native breast. Bras for native breasts must consider gravitational effects and gathering of the breast within the cup of the bra. For round implant-reconstructed breasts, the breast tissue does not move to fill the bra cup, so the cup design pattern may be shaped so that the fabric lays flat over the breast (for example, by placing evenly placed darts in the fabric composing the cup). As implants are not uniformly placed in the same location on the torso, bra straps may need to be more adjustable to accommodate for the new position of the transition point. For example, bra straps could be redesigned so as the connection between the cup and strap is adjustable to be positioned higher on the torso to improve comfort and provide proper support. The secondary symmetry measures also reveal that asymmetry increases significantly after mastectomy, even when unilateral implant reconstruction and a contralateral symmetry procedure are performed. Bras should be able to accommodate for different volumes and shapes of the right and left breasts. Patients with severe asymmetry may require a custom-made garment. Future work may investigate other factors that may impact bra design including whether the patient received radiation therapy and implant position relative to the pectoral muscle, i.e., above the pectoralis muscle (pre-pectoral) or below the pectoralis muscle (sub-pectoral). All of the patients included in this study had sub-pectoral implants, and none received radiation.

Breast reconstruction patients have complex needs for bras, stemming from the new anatomy of their breasts as well as the persistent impacts of breast cancer treatment. The findings in this study demonstrate that clinical 3D photographs can provide insight to bra design and as well as complement other qualitative studies that highlight patients' needs and preferences for bras after breast cancer treatment.

\section{References}

[1] K. L. LaBat, K. S. Ryan, and S. Sanden-Will, "Breast cancer survivors' wearable product needs and wants: a challenge to designers," International Journal of Fashion Design, Technology and Education, vol. 10, no. 3, pp. 308-319, Sep. 2017.

[2] A. L. Cheong et al., "Natural Breast Symmetry in Preoperative Breast Cancer Patients," Plastic and reconstructive surgery. Global open, Plastic and Reconstructive Surgery Global Open, vol. 7, no. 7, Jul. 2019.

[3] K. M. Nicklaus et al., "Correspondence of Breast Measurements for Bra Design After Reconstruction Surgery," presented at the Proc. of 3DBODY.TECH 2017 - 8th Int. Conference and Exhibition on 3D Body Scanning and Processing Technologies, Montreal QC, Canada, 11-12 Oct. 2017, 2017.

[4] H. Panchal and E. Matros, "Current Trends in Post-Mastectomy Breast Reconstruction," Plastic \& Reconstructive Surgery, vol. 140, no. 5S, pp. 7S-13S, Nov. 2017.

[5] J. Lee et al., "Validation of Stereophotogrammetry of the Human Torso," Breast Cancer (Auckl), vol. 5, pp. 15-25, Feb. 2011.

[6] M. M. Kawale et al., "Automated Identification of Fiducial Points on 3D Torso Images," Biomed Eng Comput Biol, vol. 5, pp. 57-68, Jul. 2013.

[7] M. Kawale et al., "3D Symmetry Measure Invariant to Subject Pose During Image Acquisition," Breast Cancer (Auckl), vol. 5, pp. 131-142, 2011.

[8] G. P. Reece et al., "3D surface imaging of the human female torso in upright to supine positions," Med Eng Phys, vol. 37, no. 4, pp. 375-383, Apr. 2015.

[9] L. Zhao, A. Cheong, G. P. Reece, M. C. Fingeret, S. K. Shah, and F. A. Merchant, "Inferior BreastChest Contour Detection in 3-D Images of the Female Torso," IEEE Journal of Translational Engineering in Health and Medicine, vol. 4, pp. 1-10, 2016.

[10]E. Van Limbergen, E. van der Schueren, and K. Van Tongelen, "Cosmetic evaluation of breast conserving treatment for mammary cancer. 1. Proposal of a quantitative scoring system," Radiotherapy and Oncology, vol. 16, no. 3, pp. 159-167, Nov. 1989. 